\title{
Ambigüedad en el léxico económico de Hamlet (1.3.88-136) y su tratamiento en la versión alemana de August Wilhelm Schlegel
}

\author{
(Ambiguity in Hamlet's economic terms 1.3.88-136 and its \\ rendering into German: August Wilhelm Schlegel's translation)
}

\author{
ALFONSO CORBACHO SÁNCHEZ/LUIS JAVIER CONEJERO MAGRO \\ alcorsan@unex.es/conejeroluis@unex.es \\ Universidad de Extremadura
}

Fecha de recepción: 27 de octubre de 2018

Fecha de aceptación: 30 de noviembre de 2018

Resumen: Como es sabido, a pesar de su complejidad textual, las obras de William Shakespeare han generado un número considerable de traducciones a las principales lenguas del mundo. En este sentido, el presente estudio tiene un doble propósito: en primer lugar, trata de identificar y analizar las ambigüedades de algunos términos usados por Shakespeare en Hamlet 1.3.88-136 y, a continuación, realiza un cotejo de la traducción alemana de August Wilhelm Schlegel con el texto original, a fin de comprobar en qué medida se recrean los diversos sentidos y los múltiples significados de estos términos en la versión alemana.

Palabras clave: Shakespeare. Hamlet. Ambigüedad. Juego verbal. Traducción. Alemán.

\begin{abstract}
As is well known, in spite of their textual complexity, the works of William Shakespeare have given rise to a considerable number of translations into the major languages of the world. The aim of this article is twofold: firstly, to identify and analyze the ambiguities of some terms used by Shakespeare in Hamlet 1.3.88-136. Then, to contrast the German translation by August Wilhelm Schlegel with the original text, in order to point out to what extent the various senses and the multiple meanings of these terms have been preserved in this German version.
\end{abstract}

Key words: Shakespeare. Hamlet. Ambiguity. Wordplay. Translation. German. 


\section{Alfonso Corbacho/ Luis JaVier CONEJERO}

\section{Introducción}

Son de sobra conocidos tanto el uso frecuente que hace Shakespeare de la ambigüedad y de los dobles sentidos de las palabras como la función que este juego verbal suele tener en sus textos ${ }^{1}$. Esa función va desde el efecto puramente cómico, que es sin duda alguna el que predomina, hasta otros de tipo más serio e incluso dramático. Pues bien, como se sabe también, la cantidad de unidades léxicas, sintagmáticas y hasta morfosintácticas marcadas por el retruécano y la ambigüedad es de tal envergadura, y su distribución tan bien calculada, que estamos sin duda ante uno de los recursos estilísticos más importantes de este autor; y no sólo, según se acaba de indicar, por el papel que desempeña en la caracterización de los personajes más importantes e incluso en la presentación e interpretación de simples anécdotas o acontecimientos célebres, sino sobre todo en la configuración del estilo literario. Todo esto resulta ya innegable hoy en día, máxime a la luz de los nuevos enfoques y la extensa bibliografía que se ha producido y se está produciendo en este sentido ${ }^{2}$. El corolario traductológico de esta nueva realidad textual es bien simple: la nueva luz que esta corriente crítico-textual ha arrojado sobre la obra de Shakespeare, en lo que supone un mayor acercamiento al significado original del texto isabelino, puede proyectarse también sobre cualquier traducción que se precie. Lo que se descubre hoy en un texto tan antiguo deben reflejarlo también sus traducciones. De ahí la ineluctabilidad de cotejar todas las traducciones existentes con esa nueva lectura de los textos isabelinos y someterlas a una crítica basada en esa luz que han arrojado los lexicólogos, analistas de textos e incluso editores de estas últimas generaciones.

Las traducciones alemanas no pueden suponer una excepción; y tal vez, al menos en el sentido y la parcela señalados, convendría hacer con ellas lo que algunos estudiosos ya han hecho con muchas de las traducciones españolas. No se trata tanto de retraducir como de señalar las deficiencias de la traducción en los textos de llegada; y, sobre todo, de presentar un texto original fiablemente interpretado a los futuros traductores de Shakespeare a la lengua alemana. Huelga decir que no es éste el objetivo del presente estudio, pero éste es el marco en que se encuadra el análisis del pasaje tan significativo que se ha seleccionado para este trabajo.

1 Véase el trabajo seminal de Empson (1930), así como los trabajos de López Ortega \& Marín Calvarro (1994), Oncins Martínez (1996), Sánchez García (1999) y Marín Calvarro (2000).

${ }^{2}$ Destacan, entre otros, los estudios de Babkoc (1985), Bather (1977), Mahood (1957, 1973), Muir (1969), Hulme (1962), O’ Hanlon (1953) y Tilley (1924). 
Ambigüedad en el léxico económico de Hamlet...

Una somera exposición de la tipología de esas ambigüedades y de la de los juegos verbales usados por este autor, así como de las funciones a que ésta sirve su obra, no sólo se saldría del cometido de este trabajo, sino que aumentaría excesivamente su extensión sin añadir nada de lo que aquí se pretende. Baste de momento con afirmar que los dobles sentidos que se analizan en estas páginas no tienen una función sencilla, pues aun incidiendo sobre lo cómico, tienen bastante de trágico. $\mathrm{Y}$, al igual que ocurre en las demás obras de Shakespeare ${ }^{3}$, también aquí, y en este pasaje concreto, los dobles sentidos contribuyen a marcar los perfiles de un personaje. En el caso del diálogo entre Polonius y su hija Ophelia contribuye a la caracterización del primer personaje, pues es tanto en las intervenciones de éste como en la manera en que él interpreta las palabras de Ophelia donde se da la ambigüedad. Dicho de otro modo, es él, y no Ophelia, quien usa la ambigüedad o ve ambigüedad donde no se genera intencionadamente. Ophelia utiliza un lenguaje convencional para expresar sus sentimientos; pero su padre, ese astuto y calculador cortesano de cuyas intrigas y personalidad retorcidas tantas páginas se han escrito, es capaz de ver bajo ese discurso normal y esas palabras inocentes los dobles sentidos de su doble moral y de sus aviesas intenciones. A lo largo del análisis, se muestra un retrato somero de Polonius, pues esto servirá para comprender el significado que esas palabras sencillas adquieren en la mente diabólica de Polonius, "a master of intrigue who controlled an elaborate network of spies", tal y como señala John Paul Stevens (1992: 1378), realizando una pertinente comparativa entre Polonius y uno de los hombres de confianza de Isabel I ${ }^{4}$.

\section{Análisis}

\subsection{Tender, True Pay y Sterling}

El primer término que cabe incluir en el léxico específico de la economía es tender(s), utilizado como sustantivo y como forma verbal, que aparece cinco veces en el pasaje seleccionado. Lo utiliza Ophelia cuando Polonius, su padre, requiere de ella que le confiese la verdad sobre su relación con Hamlet. Y la verdad es, para decirlo con las mismas palabras de ésta, que "He hath ... of late made many tenders of his affection to me" (1.3.99-100). El término reaparece cuatro veces más, en las dos intervenciones siguientes de Polonius, y en ellas lo utiliza tanto en el mismo sentido que poseía en boca de su hija -

3 Sirvan de ejemplo los trabajos ya citados de López Ortega \& Marín Calvarro (1994), Oncins Martínez (1996), Sánchez García (1999) y Marín Calvarro (2000).

${ }^{4}$ Véanse también los estudios más recientes de Holderness (2005), Schalkwyk (2005) y Caruso (2007). 


\section{Alfonso Corbacho/ Luis Javier Conejero}

en la intervención siguiente- como en sentidos marcadamente económicos -en la intervención posterior de Polonius-. En esta última, el contraste del sentido de tenders y de los sintagmas verbales "tender yourself" y "you'll tender" con respecto al que tenía en boca de Ophelia es tan marcado que se puede hablar de una antanaclasis múltiple. La marca semántica en estos tres últimos es de naturaleza económica o contable. Lo que le dice a su hija es simplemente “... you have ta'en these tenders for true pay/Which are not sterling. Tender yourself more dearly" (I.iii.106-107). Pues bien, como se acaba de indicar, en la primera de estas intervenciones de Ophelia, tender tiene el significado de algo que se ofrece para ser aceptado, según se registra en el Oxford English Dictionary (OED); y, por el contexto del pasaje y de la frase, "equivale a muestras de afecto". Idéntico sentido posee el término en las frases de la primera respuesta de Polonius a su hija ("Do you believe his tenders...?” [1.3.103]). Según se acaba de advertir también, donde el significado del término cambia radicalmente es en la siguiente intervención de Polonius (“...Think yourself a baby/That you have ta'en these tenders for true pay/Which are not sterling...” [1.3.105-107]) Aquí, como se deduce de los términos true pay y sterling, tender se utiliza también en otra de las acepciones que tenía en el siglo XVI y aún conserva hoy en el sintagma legal tender. Equivale a divisa o moneda de curso legal. Así lo interpreta, por ejemplo, Harold Jenkins (1982) en la conocida edición de Hamlet de The Arden Shakespeare, pues para él el término no sólo tiene el sentido de "expression of feeling", sino también el de "formal presentation of money in payment" (Jenkins 1982: 204). También Graham Holderness (1992: 108) glosa este segundo sentido de tender ("presentation of money"); e igualmente Susanne L. Wofford (1994: 45), para quien equivale a "money offered in payment". Se podrían citar muchos más editores y algún que otro lexicógrafo especializado en el lenguaje y la época de Shakespeare, pero no parece necesario en este caso, ya que, de uno u otro modo, los dos sentidos conservan cierta vigencia en la actualidad. Los dos usos verbales de tender en estas últimas palabras de Polonius no hacen sino corroborar ese sentido cuasi comercial. Así se deduce tanto del consejo que le da a su hija, "tender yourself more dearly" (1.3.107) como de la advertencia de que, si no lo hace así, "you'll tender me a fool" (1.3.109). En el caso de "tender yourself more dearly", la anfibología de valoración o autoestima personal, que es ya de por sí de naturaleza contable, se ve potenciada por la presencia y ambigüedad de dear/ly —ambigüedad que si bien no conserva el término español 'querido', sí se aprecia en la forma más arcaica 'caro'. En el OED aparece ya documentado el verbo tender en el sentido de 'sentir o actuar tiernamente' o 'mirar o tratar con ternura', desde 
Ambigüedad en el léxico económico de Hamlet...

finales de la Edad Media. T. J. B. Spencer (1996: 231 y sigs.) lo ilustra como "look after" y "steem for". Y en el caso de la primera aparición de tender con función verbal se produce un fenómeno similar al que se observa en el último uso de este mismo término con función sustantiva. En efecto, en el caso del verbo tender, éste sufre una modificación por el adverbio dearly; en el del sustantivo referido en la misma oración del verbo se encuentra con el sintagma true pay, y en la relativa siguiente con el adjetivo sterling. No es necesario añadir nada más sobre el sentido económico del sustantivo pay; y en cuanto al adjetivo sterling, que podía utilizarse no sólo para denotar la autenticidad del dinero sino también de cualquier otro objeto o aspecto, no cabe duda de que su uso se fue restringiendo y limitando gradualmente a contextos comerciales y bancarios. En cuanto a "... you'll tender me a fool" (1.3.109), la gama polisémica se incrementa si cabe con respecto a los usos anteriores del verbo. En concreto, para John Dover Wilson (1954: 156), la frase no significa sólo "make a fool of yourself in my eyes", sino también "present me with a baby". Este sentido de fool como niño bastardo lo recoge Wilson de la edición de Edward Dowden (1899), y se repetirá posteriormente en otras ediciones y glosarios de prestigio.

En consecuencia, se identifica tender con el primero de una serie de términos que en boca de Ophelia expresan, por una parte, un intercambio de afecto y amor, y en la de Polonius, por otra, una transacción económica fallida. Y es también, por tanto, la primera de un grupo de metáforas que equiparan las relaciones amorosas a las financieras con una función muy significativa a la hora de marcar el perfil caracterológico de cada uno de los personajes.

Este juego verbal —según el cual el padre de Ophelia parece incapaz de hablar del amor que siente su hija sin términos que no connoten lo crematístico o lo mercantil, mientras su hija los utiliza y siente en su mera denotación afectiva - va mucho más lejos aún, e inyecta una alta dosis de brutalidad al diálogo. Pues a los dobles sentidos indicados se suma otro que connota, sin dejar lugar a dudas, un comercio de tipo sexual, es decir, el mundo de la prostitución. Porque tender, utilizado como verbo, equivalía en el inglés subestándar isabelino a ofrecerse sexualmente (Rubinstein 1989: 271). En ese nuevo contexto, more dearly seguiría expresando el valor - más caropero de los servicios sexuales prestados; y lo mismo podría decirse con respecto a pay. "Pay", efectivamente, puede leerse como el pago por esos servicios sexuales; pero, como recoge Frankie Rubinstein (1989: 6) en su glosario sobre ambigüedades en Shakespeare, "pay" es también en el inglés 


\section{Alfonso Corbacho/ Luis Javier CONEJERO}

isabelino vulgar un término para referirse a las partes pudendas de la mujer, con lo que el arco de sugerencias y asociaciones se amplía aún más.

La suerte que corre en la traducción cotejada tanto el sustantivo tenders como la forma verbal tender, así como las demás formas comentadas -true pay, sterling- y, por supuesto, la expresión make a fool, es muy diversa. Así, el término tenders, en su primera aparición ("... many tenders/ Of bis affection..." [1.3.99-100]), se transforma en "Anträge [mir getan Von seiner Zuneigung]" en la traducción de A. W. Schlegel ${ }^{5}$. Según se puede observar, la forma por la que opta el traductor alemán, "Anträge", se ciñe a ese sentido simple con el que Ophelia utiliza "tenders". Lo mismo se cumple con la segunda aparición del término, esta vez en la réplica de Polonius, pues lo emplea con idéntico sentido.

Sin embargo, cuando el mismo término se hace semánticamente más complejo, en concreto en la intervención siguiente de Polonius, en la que responde de nuevo a su hija, Schlegel no logra mantener esa complejidad. Así, esa acepción crematístico-mercantil que también posee "tenders", y que es la que está utilizando Polonius (“... you have ta”en this tenders for true pay/Which are not sterling" [1.3.106-107]), se pierde por completo al repetir el mismo término utilizado por Ophelia ("Anträge"), dado que este sustantivo no posee en alemán una segunda acepción o una connotación de estas características. Con ello se pierde también la antanaclasis que se origina al repetir Polonius este término polisémico, que parte de la primera intervención de Ophelia, en un sentido bien distinto al utilizado por su hija.

Antes de seguir con el tratamiento traductológico de los sentidos que tiene tender como forma verbal en - "Tender yourself more dearly" (1.3.107) y "you'll tender me a fool" (1.3.109) — en palabras de Polonius, conviene ver la

${ }^{5}$ August Wilhelm von Schlegel (1767-1845), uno de los máximos exponentes del Romanticismo alemán, destacó no sólo como filólogo y crítico literario, sino también como traductor de un talento extraordinario, sobre todo por sus versiones ya "clásicas" de la obra de Shakespeare en lengua alemana. Como romántico, sus principios de traducción implican una gran fidelidad al sentido del original y una carga creativa significativa a la hora de producir el texto meta, alejándose de lo que supone una mera copia de la obra e intentando, además, no eliminar ni añadir nada para no mutilar el texto de partida (vid. P. Gebhardt, 1970: 105), si bien es cierto que -como se puede comprobar en este estudio— no siempre logra cumplir escrupulosamente esta última premisa. Asimismo, cabe destacar otra de sus pretensiones como fue la de "alemanizar" los escritos de Shakespeare. Con el fin de acercar al lector alemán al contenido de la obra del dramaturgo inglés, Schlegel, en su labor creativa, intentó utilizar recursos lingüísticos equivalentes en alemán que consiguieran reproducir el estilo y la estética del original. Para obtener referencias más detalladas y exhaustivas sobre el método de traducción de Schlegel, pueden consultarse las publicaciones de Gebhardt (1970) -centrada en Hamlet—, Scheu Lottgen (1995) y Scheu Lottgen/Aguado (1995). 
suerte que ha seguido en la versión alemana "true pay" y "sterling". En el caso de "true pay" Schlegel lo vierte por "bar", con lo que se mantiene en cierto modo el sentido superficial de "pay". Y permítase insistir en lo del 'sentido superficial' ya que, como se verá más adelante, tanto la carga semántica de este término como la del sustantivo tender es aún mayor. Por lo que respecta al adjetivo "sterling", — como se indicaba anteriormente, hoy en día se utiliza casi exclusivamente en contextos dinerarios, pero en la época y en la obra de Shakespeare posee además el sentido general de 'auténtico' o 'genuino'- este término se pasa totalmente por alto en el texto alemán, vertiéndose únicamente ese sentido restringido de la autenticidad del valor económico. Ése es el sentido, como se puede apreciar, de "ohn Ertrag" en el texto de Schlegel.

La forma verbal "tender yourself [more dearly]" (I.iii.107) se convierte en "betragt Euch klüger" en la versión alemana. Y aquí, como se desprende del cotejo con la frase original de Polonius, es donde se aprecia la primera pérdida de esa rica polisemia que atesora el diálogo seleccionado. En efecto, el traductor se limita a trasladar al alemán ese consejo normal e inocente de la advertencia de un padre a su hija en circunstancias de este tipo; pero se descuida esa insinuación que hace Shakespeare gracias al doble sentido del verbo "tender", ya que ni para él ni para Hamlet la situación es probablemente tan típica. Se sostenía antes que probablemente a Polonius le interesaba más la corona que un día ceñiría Hamlet que la propia persona o el amor del príncipe; y si eso es así, como parece el caso, su semejanza con la prostitución no parece tan lejana. De ahí que no tiene por qué extrañar que, desde el punto de vista de Shakespeare, como tal vez del de Hamlet, el "quiérete a ti misma más" o "valórate más alto" (pues "dearly", según se recordará, equivalía a más "caro" en el sentido afectivo y en el crematístico), que es lo que literalmente le está indicando Polonius a su hija, tengan esa insinuación de la venta del cuerpo en el mercado de la prostitución, que también connota "tender" en el inglés isabelino. Otro tanto ocurre con "or [...] you'll tender me a fool" (1.3.108-109), que Schlegel transforma en "sonst [...] trägt Eure Narrheit noch Euch Schaden ein". En esta traducción no sólo se pierde por completo esa velada alusión al hecho de que si Ophelia continúa así su relación con Hamlet terminará en estado y, por lo tanto, le dará uno de esos hijos cuya ilegitimidad tanto parece avergonzar a Polonius; sino que ni siquiera se conserva esa imagen de "parecer estúpida" o "mostrarse como una idiota" que el texto alemán posee también. Lejos de ello, Schlegel se inclina por la imagen del daño que se puede hacer a sí misma. Por consiguiente, la traducción resulta, en un sentido, no sólo excesivamente libre sino también, 


\section{Alfonso Corbacho/ Luis Javier Conejero}

en otro sentido, 'liberada' de una imagen muy elocuente, en el nivel superficial de la frase isabelina; y desprovista asimismo de esa insinuación, muy pertinente en el contexto, que se genera por el doble sentido de "fool", en el nivel más profundo de esas palabras de Polonius.

\subsection{Entreatment y Rate}

Otro término que atesora una ambigüedad muy en línea con las de los anteriores es entreatment. Aparece posteriormente, en boca de Polonius, en la frase "Set your entreatments at a higher rate..." (1.3.122). No cabe duda alguna de la connotación económica que atesora el término, y no sólo por el contexto en que se da sino también porque va seguido del sintagma "at a higher rate". El sentido primero o superficial de entreatment en este pasaje es el de "conversation" o "interview", según se registra en el OED; pero en este mismo diccionario se revela otro, "negotiations", de uso muy frecuente en el lenguaje militar y, por extensión, también en los registros de carácter políticodiplomático y económico (sobre todo, en la negociación de naturaleza financiera). Algunos autores de ediciones de Hamlet, como A. W. Verity (1947), J. D. Wilson (1954), E. Hubler (1987) y B. Lott (1997) se limitan al primero de los significados señalados, esto es, al de conversación o encuentro. Pero ya H. Jenkins (1982: 206), y posteriormente autores de la talla de G. R. Hibbard (1987), R. Gill (1997), C. Hoy (1992) y J. Markus (1993), recogen la ambigüedad de esta expresión añadiendo ese otro sentido que lo vincula con la milicia y, por tratarse de situaciones muy parejas, también con la diplomacia y hasta con el mundo mercantil. Verity (1947) y J. F. Andrews (1993) van incluso más lejos, mostrando matices adicionales de sentido que potencian aún más la carga polisémica de este término. En efecto, para Verity (1947: 149), además de "conversation" e "interview", entreatment equivale a "solicitation of you"; y para Andrews (1993: 50) equivale a "both (a) your treatment of him, your willingness to give him audience; and (b) your willingness to receive his entretalles to you [to surrender]".

En cuanto a rate ("at a higher rate"), participa de esa misma duplicidad de sentido que exhiben los términos anteriores, si bien en este caso es el primario el que es de naturaleza económica, siendo el segundo traslaticio, ya que Polonius lo utiliza metafóricamente. La idea es la de darse a valer, si bien la metáfora "at a higher rate" contiene una carga económica mayor y, si se permite, más descarada; correspondería a 'cotízate más alto'. Así pues, se presentan palabras de cierta dureza, con las que Polonius ordena a su hija no sólo que se 'dé más a valer', sino que saque más provecho de sus encuentros y conversaciones con el joven Hamlet. La dureza no sólo procede del consejo 
Ambigüedad en el léxico económico de Hamlet...

de que se 'dé más a valer', que puede resultar habitual en un padre; sino de esa carga económica, e incluso casi diplomática y hasta militar, que posee también entreatments. Y es precisamente esa riqueza polisémica de este último término la que no logra transmitirse en la versión alemana: "ein wenig mehr; schätzt Eure Unterhaltung Zu hoch". La forma "Unterhaltung", como es sabido, carece de esa gama de matices que atesora el inglés isabelino. Por el contrario, Schlegel conserva esa imagen metafórica de "set... at a higher rate", dado que "schätzt ... zu hoch" transmite la idea de valorarse más o darse más a valer, partiendo de una metáfora de tipo bancario. Esto, sin embargo, no implica que en el texto alemán permanezca esa idea subyacente en la obra isabelina, según la cual Polonius estaría casi vendiendo a su hija; y ello se debe a que al pasar al alemán desaparecen por completo esas leves — pero muy obviasinsinuaciones de prostitución que se detectan en el texto de partida.

\subsection{Brokers, Investments y Suits}

A continuación, y siempre procedentes de Polonius, se relacionan tres términos más cuya manifiesta polisemia incluye también, de manera muy patente, un perfil económico-financiero. Se trata, concretamente, de brokers, investments y suits.

Con respecto a la ambigüedad del primero, aparece resuelta en el $O E D$; ya que en ese diccionario se registran, en la época, y entre otros, dos grupos de sentidos bien diferenciados: en el primero de ellos se incluyen "a retaylor of commodities; a second-hand dealer"; y dentro de este primer grupo, los de "retaylor" y "dealer in second-hand furniture and apparel". En el segundo figuran "one employed as middleman to transact business o negotiate bargains between different merchants o individuals; "a go-between or intermediary in love affairs; a procurer, pimp; bawd; a pander generally"; y "a legal agent, a proctor". El juego que permite una gama tan variada de sentidos -que, por lo demás, guardan una innegable coherencia entre sí- es enorme; y, lo que más interesa desde la perspectiva de este análisis, incluye lo estrictamente comercial, lo judicial o legal y el tráfico o comercio sexual del mundo de la prostitución. A los editores más autorizados de Hamlet no se les escapa esta multiplicidad de sentidos que atesora el término. Así, para Wilson (1954: 264) se da un interesante juego verbal entre "(a) middle-man, comission-agent” y “(b) go between pander”. Según M. M. Mahood (1957: 119), este término también incluye un doble sentido: "go-between" y "second-hand-clothes dealer". En el trabajo de Mahood se añade un comentario muy elocuente en cuanto al significado y la función de brokers: $A$ broker, besides being a go-between, could be a second-hand-clothes dealer, and Polonius' 
disguise and mystification makes bim develop this second meaning... (Mahood 1957: 119). Y según P. Edwards (1985: 100) broker destaca también por la polisemia que confieren al sustantivo los tres sentidos siguientes [que toma de $\mathrm{N}$. Alexander (1973)]: "financier" "epander" y "an old clothes man". En las ediciones de Jenkins (1982: 206), Hibbard (1987: 179) y Andrews (1993: 50) se recogen y repiten las palabras de la glosa de Wilson (1954).

Como se puede observar, también esta gama de connotaciones y matices se pierde en la traducción aquí cotejada. Concretamente, en lo que afecta al complejo e interesante problema polisémico que plantea brokers, en la versión de Schlegel se produce una trasposición de sentidos por la que lo traslaticio y oculto de corte sicalíptico de la lectura subyacente - que no es sino esa interpretación subestándar de broker como 'chulo', 'celestino' o 'alcahuete'no sólo prevalece sobre el registro económico-financiero del nivel superficial -intermediario comercial o de otro tipo de actividad lícita- sino que lo desplaza. Eso es así porque Schlegel decide traducir broker por "Kuppler" que, como se sabe, tiene únicamente acepciones peyorativas "denn sie sind Kuppler".

Por lo que respecta a "investments" (1.3.128), también cuenta con una doble proyección semántica en inglés isabelino que guarda una gran coherencia con las anteriores y contribuye enormemente a cohesionar la doble lectura que se desprende de las palabras de Polonius; y lo mismo se cumple con el término "suits" (1.3.129) en el verso siguiente. En efecto, por lo que respecta a investments, además del sentido económico-financiero de 'inversiones', posee en aquella época el de 'vestimentas' (Mahood 1957). En concreto, en el $O E D$ se glosan, entre otros significados los de "1...clothing; robes, vestments"; y "5. The investing of money or capital". J. D. Wilson (1954: 156, 275) menciona explícitamente el juego verbal que se configura en torno al doble sentido del término que interpreta como "vestments", por una parte, y "money, investments" por otra. M. M. Mahood (1957: 119-120) y J. Jenkins (1982: 202) van más lejos en cuanto a la carga polisémica del término, añadiendo a los dos sentidos ya mencionados, otro de tipo militar, "sieges", que iría también muy en consonancia con el de entreatments; y, desde luego, guarda igualmente una gran coherencia con la gama de significados que entraña "suits". En concreto, en el OED se registran sus sentidos legales ("pursuit; persecution, legal process"); su matiz claramente económico ("a due paid in lieu of attendance at the cort of lord"); el referente al cortejo ("wooing or courting of a woman"; "solicitation of a woman's hand"); y el de "suit of apparel, of clothes". Toda esta gama de sentidos genera, como es lógico, un interesante juego polisémico que los principales editores de Hamlet 
Ambigüedad en el léxico económico de Hamlet...

recogen. Es más, algunos de ellos hacen matizaciones que potencian aún más el retruécano, desvelando y aportando nuevos matices semánticos. Así, B. Lott (1997: 32), glosa "unholy suits" como "wicked causes". Por su parte, H. Jenkins (1982: 206) se refiere también al retruécano que se establece entre "suits" e "investments". G. R. Hibbard (1987: 179) hace equivaler "suits" a "inducements (to elicit love)". Para B. A. Mowat (1992: 46), "suits" equivale a "actions"; y para Markus (1993: 58), a "deeds". Por último, D. Delabastita (1993: 366) resume las anotaciones de los principales autores que comentan la multiplicidad de sentidos que encierra el término en dos grupos bien diferenciados: por una parte, "S1: causes, requests, inducements, courtship"; y, por otra, "S2: sets of clothing".

En esta tesitura, Schlegel no parece resolver con gran acierto los problemas de traducción que plantea el original isabelino. Así, al verter los versos "Not of that dye which their investments show,/But mere implorators of unholy suits..." por "Nicht von der Farbe ihrer äußern Tracht, Fürsprecher sündlicher Gesuche...", pierde por completo la rica polisemia que entrañan "investments" y "suits". En efecto, el sustantivo "Tracht", en el contexto en el que lo utiliza, reduce su significado al de indumentaria o vestido; y "Gesuch" al de petición, solicitud, demanda o instancia. De esta forma, no sólo se les da a estos términos un valor monosémico, sino que se destruye la coherencia o, si se prefiere, ese paralelismo bisémico que mantienen los términos originales entre sí, e incluso con algunos de los demás términos ambiguos del pasaje.

\section{Conclusiones}

Como se ha podido comprobar, a lo largo del análisis de este reducido corpus de segmentos ambiguos oportunamente distribuidos por el dramaturgo isabelino en el diálogo que sostiene Polonius con su hija Ophelia, el texto del traductor alemán que se ha cotejado con el original shakespeariano desentona con dicho original. Y en esto no puede haber más coincidencia con los trabajos de investigación referidos a las versiones españolas $^{6}$. La práctica totalidad de esos estudios demuestra que los traductores no advierten, o simplemente no son capaces de verter, todo ese entramado de retruécanos, dilogías, anfibologías y juegos verbales, en general,

${ }^{6}$ De hecho, a una conclusión muy parecida se llega en los estudios textuales y traductológicos realizados por José Luis Oncins Martínez (1996) — con respecto a las traducciones españolas de Timon of Athens - y Manuel Sánchez García (1999) — con respecto a The Taming of the Shrew y sus traducciones-; por no citar otros estudios, aunque de menor extensión, pero idéntica profundidad. 


\section{Alfonso Corbacho/ Luis Javier Conejero}

que subyace bajo el texto shakespeariano. Esta frustración generalizada que se observa en las traducciones españolas, que también se da en la versión alemana referida a la muestra aquí analizada y cotejada, se presta a una serie de matizaciones. En concreto, se aprecia cierto grado de desviación y alejamiento del texto de llegada con respecto al original indicado.

Así pues, si bien es cierto que Schlegel, según se ha podido comprobar a lo largo del cotejo, deja, por un lado, bastante que desear al trasladar el componente dilógico a la lengua alemana, también saltan a la vista, por otro lado, los aciertos estilísticos del traductor alemán, sobre todo en lo que atañe a la elección de algunos términos, que por lo general arrastran cierta carga poética.

Dejar constancia detallada de los extremos que se acaban de indicar no parece un ejercicio baladí. Pues si bien la distancia que hay entre la traducción de Schlegel y el texto isabelino de partida —en lo que respecta a un componente tan importante en la configuración del tema de la obra y de su estilo como es el rico entramado de juego verbal al que de modo tan insistente se ha hecho referencia- no la hace merecedora de una recepción digna del texto shakespeariano en la lengua alemana; al margen de los aciertos que también contienen, y de los que se ha dejado constancia.

Este ensayo se ha circunscrito únicamente al juego verbal polisémico de un pasaje de la obra y, sin embargo, en estas conclusiones se hacen valoraciones de tipo general sobre la totalidad de la traducción. Lógicamente, si no se tratase de un componente que afecta tan profundamente tanto a la forma estilística como al sentido argumental de Hamlet $-\mathrm{O}$ al menos, centrado en el pasaje analizado, a la configuración de los personajes de Polonius y Ophelia—, no se extraería tamaña conclusión.

Así pues, en el supuesto más que probable de que sea la totalidad de esta tragedia de Shakespeare, Hamlet, la que haya sufrido la mutilación que a buen seguro se observa en el fragmento aquí seleccionado y analizado, habría que afirmar que la lengua alemana debe contar con una traducción de mayor solvencia de este texto clásico de las letras isabelinas. Esto, por supuesto, no quiere decir en absoluto que la versión de Schlegel carezca por completo de calidad lingüística y valor. Por el contrario, esta traducción supone un hito en el camino que ha de conducir a una versión más o menos fiel a esa variedad y riqueza estilística con que se arropa el contenido apasionante de la historia que Shakespeare narra en esta tragedia. Que constituye un hito en el camino que lleva a una versión medianamente digna del texto a la lengua alemana quiere decir que atesora elementos y fórmulas muy válidas que, en su conjunto, contribuirá al éxito de esa traducción que supondrá una recepción 
Ambigüedad en el léxico económico de Hamlet...

digna en el alemán. Pero a esa suma de aciertos individuales habrá que añadir soluciones imaginativas de esas dilogías concatenadas y estratégicamente distribuidas en el texto que deparan una segunda lectura o, si se prefiere, ese texto alternativo que complementa y completa al texto de la primera lectura. Los dos niveles, el superficial y el subyacente, suponen la lectura cabal del texto; por lo que, reducirlos simplemente a uno de los dos en la traducción es una mutilación artística, pero carente de todo arte, y traductológicamente inadmisible.

\section{Referencias bibliográficas}

AleXAnDer, Nigel (ed.), Hamlet. London: MacMillan, 1973.

ANDREWS, John F. (ed.), Hamlet. London/Vermont: Everyman, 1993.

ANDREWs, Richard; GIBSON, Rex (eds.), Hamlet. Cambridge/New York: Cambridge University Press, 1994.

BABKOC, C. M., Shakespeare: Wisdom and Wit. Mount Vernon, New York: Peter Pauper Press, 1985.

Bather, F.A., "The Puns of Shakespeare”. En: Crosbie, J.S. (ed.), Crosbie's CARuso, Carl, "Sacred Pearls in the Machinery of Hamlet". En: The Oxfordian, 10, 2007, pp. 85-110.

Dictionary of Puns, New York: Harmony Books, 1977, pp. 267-278.

DelabastiTA, Dirk, There's a Double Tongue. An Investigation into the Translation of Shakespeare's Wordplay with Special Reference to Hamlet. Amsterdam/Atlanta: Rodopi, 1993.

DowDEn, Edward (ed.), Hamlet. London: Methuen, 1899. (Arden Shakespeare)

Edwards, Philip (ed.), Hamlet, Prince of Denmark. Cambridge: Cambridge University Press, 1985.

EMPSON, William, Seven Types of Ambiguity. London: Random House, 1930.

GeBHARDT, Peter, A. W. Schlegels Shakespeare-Übersetzung. Untersuchungen zи seinem Übersetzungsverfahren am Beispiel des Hamlet. Göttingen: Vandenhoeck \& Ruprecht, 1970.

GILL, Roma (ed.), Hamlet. Oxford: Oxford University Press, 1997.

HiBBARD, George Richard (ed.), Hamlet. Oxford/New York: Oxford University Press, 1987.

Holderness, Graham; Loughrey, Bryan (eds.), The Tragical Historie of Hamlet, Prince of Denmark. Herfordshire: Harvester Wheatsheaf, 1992.

Holderness, Graham, "Vanishing Point: Looking for Hamlet". En: Shakespeare, 1, 1 y 2, 2005, pp. 154-173.

HoY, Cyrus (ed.), Hamlet. New York/London: Norton \& Company, 1992. 
Hubler, Edward (ed.), The Tragedy of Hamlet. Prince of Denmark. New York/London: Signet Classic, 1987.

Hulme, H. M., Explorations in Shakespeare's Language. Some Problems of Word Meaning in the Dramatic Text. New York: Longman, 1962.

JENkINS, Harold (ed.), Hamlet. Walton-on-Thames: Thomas Nelson \& Sons, 1982. (The Arden Shakespeare)

LÓPEZ OrTegA, Ramón; MARÍN CALvarro, Jesús, "Ambivalencia y paradoja en el Sonnet CXXXVIII de Shakespeare. Notas para su traducción”. En: Miscel-lánia D’Homenatge al Dr. Esteve Pujals. Barcelona: Columna Ediciones, 1994, pp. 192-204.

LOTT, Bernard (ed.), Hamlet. Harlow: Longman Group, 1997.

MAHOOD, Molly Maureen, Shakespeare's Wordplay. London: Methuen \& Co., 1957.

MARÍN CALVARro, Jesús, El discurso ambiguo de los diecisiete primeros sonetos de William Shakespeare y su traducción al español. Cáceres: Universidad de Extremadura, 2000.

MARKUS, Julia; JORDAN, Paul (eds.), Hamlet. Longman Literature Shakespeare, 1993.

MowAT, Barbara A.; Werstine, Paul (eds.), Hamlet. New York: Washington Square Press, 1992.

MuIR, Kenneth, "The Uncomic Pun”. En: Cambridge Journal, III, 1969, pp. 472-485.

OED, Oxford Englisch Dictionary. Oxford: Clarendon Press, 1989.

O’ HANLON, R. L., “Shakespeare’s Pun”. En: Shakespeare Newsletter, 1953, p.15.

OnCINS MARTÍNEZ, José Luis, Análisis textual y traductológico de Timon of Athens. Cáceres: Universidad de Extremadura, 1996.

RUBINSTEIN, Frankie, A Dictionary of Shakespeare's Sexual Puns and Their Significance. London: Macmillan, 1989.

SÁNCHEZ GARCÍA, Manuel, Estudio textual y traductológico de The Taming of the Shrew. Cáceres: Universidad de Extremadura, 1999.

SCHALKWYK, David, "Shakespeare's Ghosts”. En: Shakespeare, 1, 1 y 2, 2005, pp. 219-240.

SCHEU LOTTGEN, Dagmar, Duodécima noche de W. Shakespeare: traducciones al alemán por A. W. Schlegel y F. Gundolf. Estudio comparativo. Murcia: Universidad de Murcia, 1995.

SCHEU LotTGEN, Dagmar; AguAdo GimÉnEZ, Pilar, “A. W. Schlegel: los principios de fidelidad y agilidad estilística en la traducción de William Shakespeare". En: Cuadernos de Filología Inglesa, 4, 1995, pp. 75-92. 
Ambigüedad en el léxico económico de Hamlet...

SHAKESPEARE, William, Shakespeare's dramatische Werke. Berlin: Unger, 1798. (Traducción de August Wilhelm Schlegel)

Spencer, T. J. B. (ed.), Hamlet. London: Penguin Books, 1996.

Tilley, Morris Palmer, "Puns and Proverb as Aid to Unexplained Shakespearean Jests". En: Studies in Philology, 21, 3, 1924, pp. 492-495.

VERITY, A. W. (ed.), Hamlet. Cambridge: Cambridge University Press, 1947.

WiLSON, John Dover (ed.), The Tragedy of Hamlet, Prince of Denmark.

Cambridge: Cambridge University Press, 1954.

WofFOrD, Susanne L., Hamlet. Boston: Bedford Books, 1994. 
Alfonso Corbacho/ LuIS JAVIER CONEJERO

\section{Anexo}

LORD POLONIUS

What is't, Ophelia, he hath said to you?

OPHELIA

So please you, something touching the Lord Hamlet.

LORD POLONIUS

Marry, well bethought.

'Tis told me, he hath very oft of late

Given private time to yo, and you yourself

Have of your audience been most free and bounteous.

If it be so - as so 'tis put on me,

And that in way of caution - I must tell you

You do not understand yourself so clearly

As it behoves my daughter and your honour.

What is between you? Give me up the truth.

OPHELIA

He hath, my lord, of late made many tenders

Of his affection to me.

LORD POLONIUS

Affection? Pooh, you speak like a green girl,

Unsifted in such perilous circumstance.

Do you believe his tenders, as you call them?

OPHELIA

I do not know, my lord, what I should think.

LORD POLONIUS

Marry, I'll teach you. Think yourself a baby

That you have ta'en these tenders for true pay

Which are not sterling. Tender yourself more dearly

Or - not to crack the wind of the poor phrase,

Running it thus - you'll tender me a fool.

OPHELIA

My lord, he hath importuned me with love

In honourable fashion.

LORD POLONIUS

Ay, fashion you may call it. Go to, go to.

OPHELIA

And hath given countenance to his speech, my lord,

With almost all the holy vows of heaven.

LORD POLONIUS 
Ambigüedad en el léxico económico de Hamlet...

Ay, springes to catch woodcocks! I do know,

When the blood burns, how prodigal the soul

Lends the tongue vows. These blazes, daughter,

Giving more light than heat, extinct in both

Even in their promise, as it is a-making,

You must not take for fire. From this time

Be somewhat scanter of your maiden presence,

Set your entreatments at a higher rate

Than a command to parley. For Lord Hamlet,

Believe so much in him that he is young

And with a larger tether may he walk

Than may be given you. In few, Ophelia,

Do not believe his vows; for they are brokers

Not of that dye which their investments show

But mere implorators of unholy suits,

Breathing like sanctified and pious bawds

The better to beguile. This is for all.

I would not, in plain terms, from this time forth

Have you so slander any moment leisure

As to give words or talk with the Lord Hamlet.

Look to't, I charge you. Come your ways.

OPHELIA

I shall obey, my lord.

Exeunt.

(I.iii.88-136) ${ }^{7}$

(7) Edición de H. Jenkins (1982) 